\title{
Effect of a therapeutic exercise program (FisioPausa) on the quality of life of employees from CESPU
}

\begin{abstract}
Introduction In the context of work, labouring time is mainly spent in the sitting position and in a continuous way. This reality contributes for the increase of a sedentary lifestyle of workers, leading to health-related diseases and consequently decreasing they quality of life (QoL).
\end{abstract}

Objectives Verify the effect of a therapeutic exercise program in quality of life, and evaluate the association between different components of QoL, with back pain, physical activity and Body Mass Index (BMI).

Methodology A pré-post interventional study was realized in workers from the Cooperativa de Ensino Superior Politécnico Universitário. Fourteen participants were included and submitted to evaluations with a preliminary survey, the SF-36v2 (before and after 8 weeks of intervention) to assess the effects of the program in QoL. All the participants were submitted to a specific therapeutic exercise program of 20 minutes, twice a week, and during 8 weeks.

Results There was a general improvement in Health-Related Physical and Mental Quality of Life. These improvements were especially observed in the functional capacity with a higher median of $5.00(p=0,015)$. Although it was less evident, Vitality was the domain with higher improvements amongst all the four Mental domains (increase of 10.00 points across time, $p=0.341$ ). Associations were observed between pain and functional capacity $(p=$ $0,027) \mathrm{BMI}$ and Mental Health $(\mathrm{p}=0,027)$ and finally between Age and Mental Health $(\mathrm{p}=$ $0,028)$.

Conclusion Our therapeutic exercise program seems to have positive effect on quality of life, especially regarding functional capacity of workers. Participants with the highest pain 
were associated with decreased functional capacity and older people or those with higher BMI showed weaker mental health. 